\title{
Can We Have a Second Light Higgs Boson in the LHC Data?
}

\author{
Shaaban Khalil ${ }^{1}$ and Stefano Moretti ${ }^{*}$ \\ ${ }^{1}$ Center for Fundamental Physics, Zewail City of Science and Technology, Sheikh Zayed,12588, Giza, Egypt \\ ${ }^{2}$ School of Physics and Astronomy, University of Southampton, Highfield, Southampton SO17 1BJ, UK \\ Email: s.moretti@soton.ac.uk
}

\begin{abstract}
A second light Higgs boson, with mass $\approx 140-145 \mathrm{GeV}$, is predicted by non-minimal Supersymmetric models. This new particle can account for $\sim 3 \sigma$ excesses recorded by the ATLAS and CMS experiments at the Large Hadron Collider (LHC) during Run 1. We show how this can be explained in a particular realisation of these scenarios, the $(B-L)$ Supersymmetric Model (BLSSM), which also has other captivating features, like offering an explanation for neutrino masses and releaving the small hierarchy problem of the Minimal Supersymmetric Standard Model (MSSM).
\end{abstract}

In the absence of any new physics signal at the LHC above and beyond the Standard Model (SM), as it is presently the case, it has become all the more important to understand the true nature of the Higgs boson discovered in 2012. In fact, as we shall argue below, there is no need to assume that Nature prefers the minimal version of the Higgs mechanism for mass generation. Upon dismissing this assumption, alongside precision measurements of the $125 \mathrm{GeV}$ resonance, it becomes equally important to pursue the search for additional Higgs boson states. In the attempt to discern which amongst the models providing these is the true one, we believe that guidance should be taken from data. While rumours of potential heavy Higgs resonances at $\sim 750 \mathrm{GeV}$ are pervading the collider physics world, based on $\sim 3 \sigma$ excesses seen by both ATLAS and CMS, we would like to note here that comparable hints for a $140 \mathrm{GeV}$ or so additional Higgs state have been recorded on data by both collaborations with a similar level of significance and, quite crucially, not only in $\gamma \gamma$ final states (as is the case for the $750 \mathrm{GeV}$ anomaly), but also in the (more precise) $Z Z^{*}$ channel. It is our purpose here to provide a unified solution to the 125 $\mathrm{GeV}$ discovery and $140 \mathrm{GeV}$ anomaly in a next-to-minimal Supersymmetric model, which, other than inheriting the well known benefits of Supersymmetry (SUSY), can also boast theoretical attractiveness well beyond the Higgs sector. Should a $140 \mathrm{GeV}$ signal be finally confirmed by additional data, Higgs physics would be drastically advanced in the direction of pointing to non-minimal realisations of SUSY. But, let us proceed in steps.

The paradigm that particle physics is minimalist in Nature, at least as far as Electro-Weak Symmetry Breaking (EWSB) goes [1], may simply be the result of early appearance if one realises that the Standard Model (as we know it) appeared to be so initially, but then it revealed itself rather more articulated than we thought or hoped. As for its interactions, there was first the photon, uncharged and massless. We later discovered its massive weak companions, $Z$ and $W^{ \pm}$, the latter being charged too. Even the gluon is one of eight, actually. Concerning matter, the story started with one generation of quarks and leptons/neutrinos, that was sufficient to keep our world stable. Then somebody ordered the muon [2] and all apparently fell apart. With it also came its neutrino (not that we saw it at the time or even now). Strangely yet charmingly, the quarks were no less zealous, producing their own second generation of two offsprings within a few decades. Discovering the third generations of fermions was even more upsetting, so bulky in comparison to the preceding two.

We may then have to dismiss a minimalist attitude also for the Higgs sector, eventually. Intriguingly, in fact, in the search for Higgs bosons during Run 1, at $\sqrt{s}=7,8 \mathrm{TeV}$, the CMS collaboration also found potential signals for another Higgs boson, $h^{\prime}$, with mass $m_{h^{\prime}} \gtrsim 140 \mathrm{GeV}$ in the $h^{\prime} \rightarrow Z Z^{*} \rightarrow 4 l(l=e, \mu)$ decay mode [3], wherein a $\sim 2 \sigma$ excess is appreciable in the vicinities of $140-145 \mathrm{GeV}$, and in the $h^{\prime} \rightarrow \gamma \gamma$ decay channel, wherein the local $p$-value indicates possibly significant excesses very near both 140 and $145 \mathrm{GeV}$ at the $\sim 2.9$ and $\sim 2 \sigma$ level, respectively [4]. Some deviations around 140-142 GeV from SM predictions also appear in di-photon data from ATLAS [5,6]. Further, various anomalies for a mass $\gtrsim 140$ $\mathrm{GeV}$ or above have emerged in several other channels, from both ATLAS and CMS at the LHC as well as both CDF and D0 at the Tevatron [7]. It is especially worth noting that current Run 2 analyses at 
the LHC, with $\sqrt{s}=13 \mathrm{TeV}$, focused mainly on searches for heavy Higgs bosons with mass from just below $200 \mathrm{GeV}$ up to $2.5 \mathrm{TeV}$ or so, see, e.g., Refs. [8,9]. (In fact, when they do go down to $130 \mathrm{GeV}$ or so, like CMS in [10], the situation remains inconclusive.) Therefore, this Run 1 excess around 140-145 $\mathrm{GeV}$ remains unexplained to this day.

We ought to be prepared this time, then, for a non-minimal Higgs sector. SUSY, for example, calls for it. We are rather fond of this ultimate (potential) symmetry of Nature in fact, as for the first time a theory would probably solve more problems that it could create. In particular, SUSY, whichever shape or form of it is actually realised in Nature, wants a light Higgs boson, with mass similar to that of the weak gauge bosons. (No such a claim can be made by the SM instead.) Alas, SUSY has not been seen, yet. While disturbing per se, this fact may actually be a consequence of (yet again) a flawed approach, that assumes that SUSY is also minimal. Just like the SM actually is not for most of its parts (and we claim it to be for none), SUSY needs not be so either. Unsurprisingly, if one dismisses minimalism in SUSY, one may find at the same time an explanation for the absence of its manifestations at present ${ }^{1}$ as well as a hint of where Higgs companions might be. It is also worth mentioning here that in the BLSSM (with inverse seesaw) the so-called small hierarchy problem of the MSSM, i.e., the discovered Higgs boson mass of $125 \mathrm{GeV}$ being dangerously close to its predicted upper limit $(\approx 130 \mathrm{GeV})$, is relieved by providing (s)neutrino mass corrections which can up-lift this value to $\approx 170 \mathrm{GeV}[11]$.

It is remarkable though that an explanation for a second light Higgs particle cannot be found inits minimal version, the MSSM. Although it has an additional neutral and CP-even Higgs boson, it cannot account for the possibility of the aforementioned possible double Higgs peak, with masses at $\approx 125$ and $\approx 145 \mathrm{GeV}$. Furthermore, the solid experimental evidence for neutrino oscillations, pointing towards non-vanishing neutrino masses, hints at favouring other SUSY realisations than the MSSM. For example. a minimal extension of the latter is based on the gauge group $S U(3)_{C} \times S U(2)_{L} \times U(1)_{Y} \times U(1)_{B-L}$. In the ensuing model, the aforementioned BLSSM, the $(B-L)$ symmetry breaking is related to the soft SUSY breaking scale, i.e., $\mathcal{O}(1) \mathrm{TeV}$. In this class of models, with $\mathrm{TeV}$ scale right-handed neutrinos, light neutrino masses can be naturally implemented through the inverse see-saw mechanism [12]. The particle content of this model includes the following Superfields in addition to those in the MSSM: two SM singlet chiral Higgs Superfields $\hat{\chi}_{1,2}$, with the Vacuum Expectation Values (VEVs) of their scalar components $v_{1}^{\prime}$ and $v_{2}^{\prime}$ spontaneously breaking the $(B-L)$ symmetryy and with $\hat{\chi}_{2}$ necessarily required to cancel the $U(1)_{B-L}$ anomaly; three sets of SM singlet chiral Superfields $\hat{\nu}_{i}, \hat{s}_{1_{i}}, \hat{s}_{2_{i}}(i=1,2,3)$, to implement the inverse see-saw mechanism (also needed to cancel the aforementioned anomaly). The Superpotential of this model is given by [12]

$$
\begin{aligned}
W & =-\mu_{\eta} \hat{\chi}_{1} \hat{\chi}_{2}+\mu \hat{H}_{u} \hat{H}_{d}+\mu_{s} \hat{s}_{2} \hat{s}_{2}-Y_{d} \hat{d} \hat{q} \hat{H}_{d} \\
& -Y_{e} \hat{e} \hat{l} \hat{H}_{d}+Y_{u} \hat{u} \hat{q} \hat{H}_{u}+Y_{s} \hat{\nu} \hat{\chi}_{1} \hat{s}_{2}+Y_{\nu} \hat{\nu} \hat{l} \hat{H}_{u} .
\end{aligned}
$$

To obtain the masses of the physical neutral Higgs bosons in the BLSSM with inverse seesaw, one makes the usual redefinition of the Higgs fields, i.e., $H_{1,2}^{0}=\frac{1}{\sqrt{2}}\left(v_{1,2}+\sigma_{1,2}+i \phi_{1,2}\right)$ and $\chi_{1,2}^{0}=\frac{1}{\sqrt{2}}\left(v_{1,2}^{\prime}+\sigma_{1,2}^{\prime}+i \phi_{1,2}^{\prime}\right)$, where $\sigma_{1,2}=\operatorname{Re} H_{1,2}^{0}, \phi_{1,2}=\operatorname{Im} H_{1,2}^{0}, \sigma_{1,2}^{\prime}=\operatorname{Re} \chi_{1,2}^{0}$ and $\phi_{1,2}^{\prime}=\operatorname{Im} \chi_{1,2}^{0}$. The real parts correspond to the $\mathrm{CP}$-even Higgs bosons and the imaginary parts correspond to the CP-odd Higgs bosons. The squared-mass matrix of the CP-even neutral Higgs fields at tree level, in the basis $\left(\sigma_{1}, \sigma_{2}, \sigma_{1}^{\prime}, \sigma_{2}^{\prime}\right)$, is given by

$$
M^{2}=\left(\begin{array}{cc}
M_{h H}^{2} & M_{h h^{\prime}}^{2} \\
M_{h h^{\prime}}^{2^{T}} & M_{h^{\prime} H^{\prime}}^{2}
\end{array}\right)
$$

where $M_{h H}^{2}$ is the usual MSSM neutral CP-even Higgs mass matrix, which leads to the SM-like Higgs boson with mass, at one loop level, of order $125 \mathrm{GeV}$ and a heavy Higgs boson with mass $m_{H} \sim m_{A} \sim \mathcal{O}(1$ $\mathrm{TeV})$. In this case, the BLSSM matrix $M_{h^{\prime} H^{\prime}}^{2}$ is given by

$$
M_{h^{\prime} H^{\prime}}^{2}=\left(\begin{array}{cc}
m_{A^{\prime}}^{2} c_{\beta^{\prime}}^{2}+g_{B L}^{2} v_{1}^{\prime 2} & -\frac{1}{2} m_{A^{\prime}}^{2} s_{2 \beta^{\prime}}-g_{B L}^{2} v_{1}^{\prime} v_{2}^{\prime} \\
-\frac{1}{2} m_{A^{\prime}}^{2} s_{2 \beta^{\prime}}-g_{B L}^{2} v_{1}^{\prime} v_{2}^{\prime} & m_{A^{\prime}}^{2} s_{\beta^{\prime}}^{2}+g_{B L}^{2} v_{2}^{\prime 2}
\end{array}\right),
$$

\footnotetext{
${ }^{1}$ Sparticle signals may undergo longer cascades in non-minimal SUSY, thus escaping usual SUSY searches.
} 


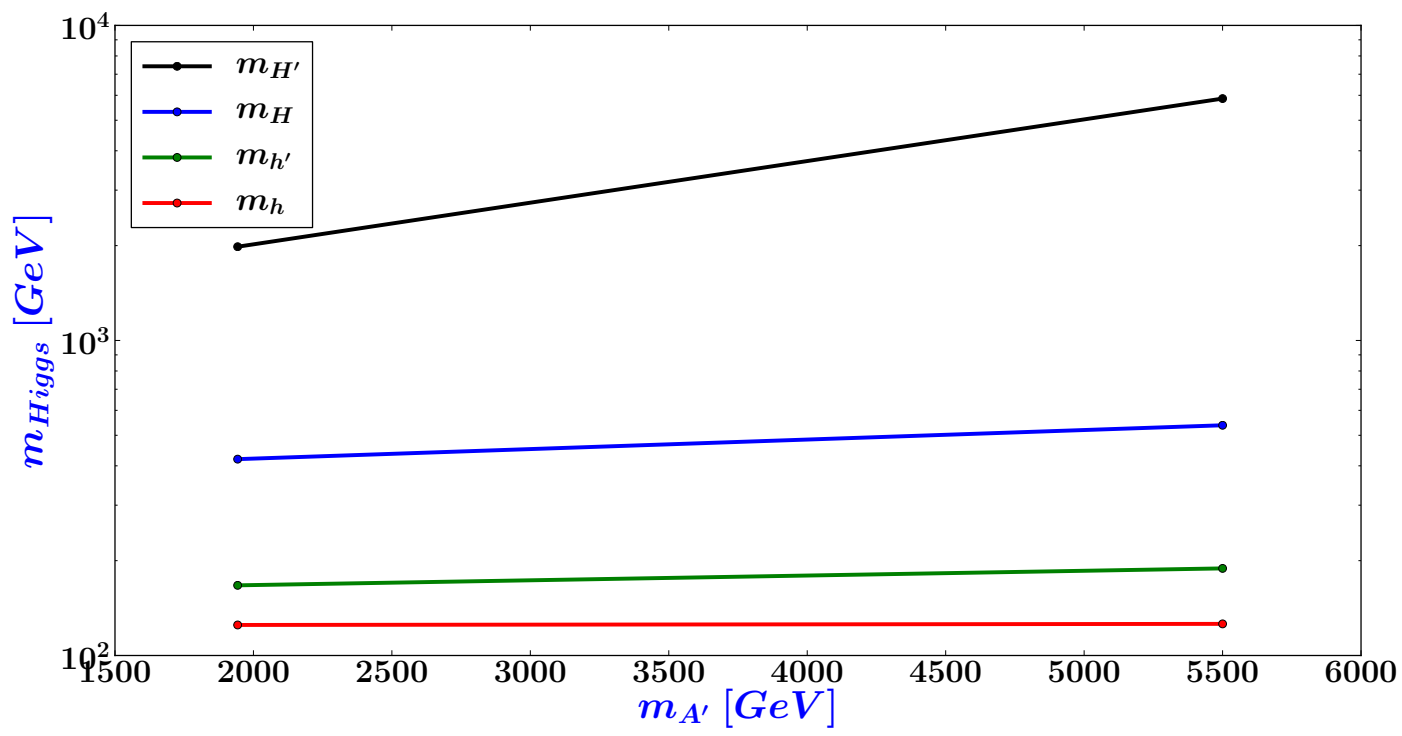

Figure 1. The BLSSM (with inverse seesaw) CP-even Higgs masses versus $m_{A^{\prime}}$ for $g_{B L}=0.55$ and $\tilde{g}=0.31$.

where $c_{x}=\cos (x)$ and $s_{x}=\sin (x)$. Therefore, the eigenvalues of this mass matrix are given by

$$
\begin{aligned}
m_{h^{\prime}, H^{\prime}}^{2} & =\frac{1}{2}\left[\left(m_{A^{\prime}}^{2}+M_{Z^{\prime}}^{2}\right)\right. \\
& \left.\mp \sqrt{\left(m_{A^{\prime}}^{2}+M_{Z^{\prime}}^{2}\right)^{2}-4 m_{A^{\prime}}^{2} M_{Z^{\prime}}^{2} \cos ^{2} 2 \beta^{\prime}}\right],
\end{aligned}
$$

where $A^{\prime}$ is a CP-odd companion of the CP-even states $h^{\prime}$ and $H^{\prime}, Z^{\prime}$ is the gauge boson associated to the spontaneous breaking of $U(1)_{B-L}$ and $\tan \beta^{\prime}=v_{1}^{\prime} / v_{2}^{\prime}$. If $\cos ^{2} 2 \beta^{\prime} \ll 1$, one finds that the lightest $B-L$ neutral Higgs state mass is given by

$$
m_{h^{\prime}} \simeq\left(\frac{m_{A^{\prime}}^{2} M_{Z^{\prime}}^{2} \cos ^{2} 2 \beta^{\prime}}{m_{A^{\prime}}^{2}+M_{Z^{\prime}}^{2}}\right)^{\frac{1}{2}} \simeq \mathcal{O}(100 \mathrm{GeV})
$$

The mixing matrix $M_{h h^{\prime}}^{2}$ is a function of the additional Higgs VEVs, $\tilde{g}$ as well as $g_{B L}$ (two gauge coupling matrix elements entering the $U(1)_{Y}$ and $U(1)_{B-L}$ kinetic mixing in the covariant derivative, see Eqs. (19)-(22) of [12]) and can be written as [13]

$$
M_{h h^{\prime}}^{2}=\frac{1}{2} \tilde{g} g_{B L}\left(\begin{array}{cc}
v_{1} v_{1}^{\prime} & -v_{1} v_{2}^{\prime} \\
-v_{2} v_{1}^{\prime} & v_{2} v_{2}^{\prime}
\end{array}\right) .
$$

For a gauge coupling $g_{B L} \sim|\tilde{g}| \sim \mathcal{O}(0.5)$, these off-diagonal terms are about one order of magnitude smaller than the diagonal ones. However, they are still crucial for generating interaction vertices between the genuine BLSSM Higgs bosons and the MSSM-like Higgs states. Note that the mixing gauge coupling constant, $\tilde{g}$, is a free parameter that can be positive or negative [13].

In Fig. 1, we show the masses of the four CP-even Higgs bosons in the BLSSM for $g_{B L}=0.4$ and $\tilde{g}=-0.4$. In this plot we fix the lightest MSSM Higgs boson mass to be of order $125 \mathrm{GeV}$. As can be seen from this figure, as intimated, one of the BLSSM Higgs bosons, $h^{\prime}$, can be the second lightest Higgs boson $(\sim 140 \mathrm{GeV})$. Both $H$ and $H^{\prime}$ are instead quite heavy (since both $m_{A}$ and $m_{A^{\prime}}$ are of order TeV).

This sets the stage for the hypothesis made in Ref. [14] (see also [15]), wherein, motivated by a $\sim 2.9 \sigma$ excess recorded by the CMS experiment at the LHC around a mass of order $\sim 140 \mathrm{GeV}$ in $Z Z^{*} \rightarrow 4 l$ 


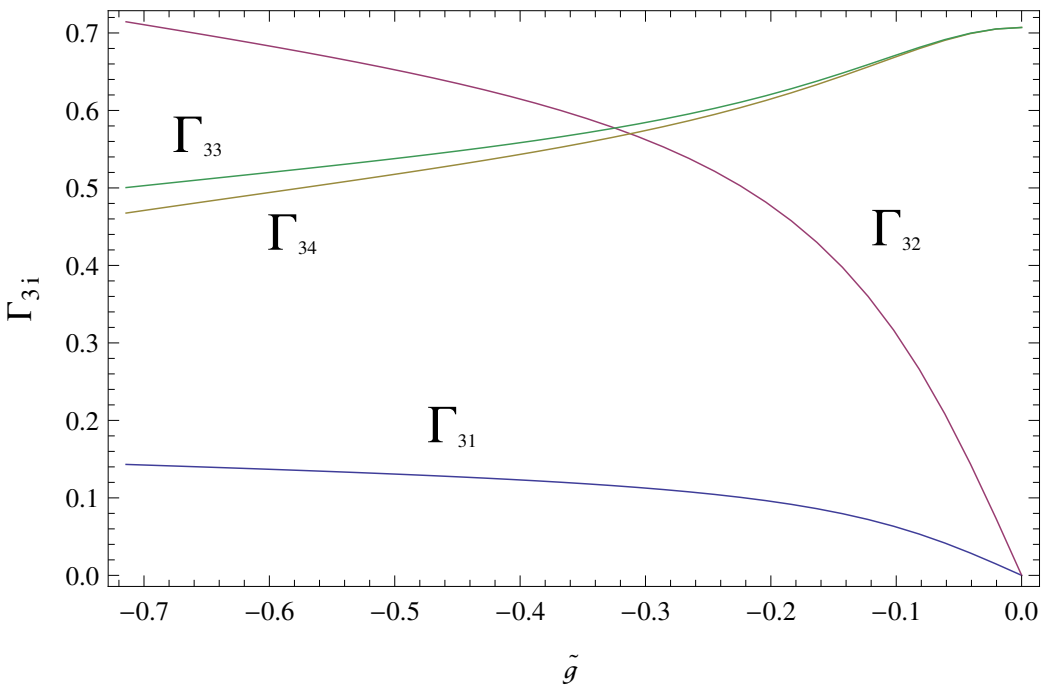

Figure 2. The mixing of $h^{\prime}, \Gamma_{3 i}$, vs the gauge kinetic one, $\tilde{g}$.

and $\gamma \gamma$ samples, is was shown that a double Higgs peak structure can be generated in the BLSSM, with CP-even Higgs boson masses at $\sim 125$ and $\sim 140 \mathrm{GeV}$, a possibility instead precluded to the MSSM.

Before proceeding in this respect, though, two remarks are in order: firstly, if $\tilde{g}=0$, the coupling of the BLSSM lightest Higgs state, $h^{\prime}$, with the SM particles will be significantly suppressed $\left(\leq 10^{-5}\right.$ relative to the SM strength), so that, in order to account for possible $h^{\prime}$ signals at the LHC, this parameter ought to be sizable; secondly, in both cases of vanishing and non-vanishing $\tilde{g}$, one may fine-tune the parameters and get a light $m_{A}$, which leads to a MSSM-like CP-even Higgs state, $H$, with $m_{H} \sim 140 \mathrm{GeV}$. However, it is well known that in the MSSM the coupling $H Z Z$ is suppressed with respect to the corresponding one of the SM-like Higgs particle by one order of magnitude due to the smallness of $\cos (\beta-\alpha)$, where $\sin (\beta-\alpha) \sim 1$. In addition, the total decay width of $H$ is larger than the total decay width of the SM-like Higgs, $h$, by at least one order of magnitude, because it is proportional to $(\cos \alpha / \cos \beta)^{2}$, which is essentially the square of the coupling of $H$ to the bottom quark. Therefore, the MSSM-like heavy Higgs signal $\left(p p \rightarrow H \rightarrow Z Z^{*} \rightarrow 4 l\right.$ ) has a very suppressed cross section and thus cannot be a candidate for light Higgs signals at the LHC.

In the light of this, we will focus in the remainder on the lightest BLSSM CP-even Higgs, $h^{\prime}$, as a possible candidate for the second Higgs peak seen by CMS in Ref. [4] in $Z Z^{*} \rightarrow 4 l$ while also checking its compatibility with the ATLAS data in $\gamma \gamma$ of Ref. [5]. However, before doing so, we ought to setup appropriately the BLSSM parameter space, in order to find such a solution. Herein, for definiteness, we consider $m_{h^{\prime}}=142 \mathrm{GeV}$ as reference BLSSM point (for which $\tilde{g}=0.31$ ). The lightest eigenstate $h$ is the SM-like Higgs boson, for which we will fix its mass to be exactly $125 \mathrm{GeV}$.

As emphasised above, in the BLSSM, it is quite natural to have two light CP-even Higgs bosons, $h$ and $h^{\prime}$, with mass $125 \mathrm{GeV}$ and $\gtrsim 140 \mathrm{GeV}$, respectively. The CP-even neutral Higgs mass matrix in Eq. (2) can be diagonalised by a unitary transformation:

$$
\Gamma M^{2} \Gamma^{\dagger}=\operatorname{diag}\left\{m_{h}^{2}, m_{H}^{2}, m_{h^{\prime}}^{2}, m_{H^{\prime}}^{2}\right\} .
$$

The mixing couplings $\Gamma_{32}$ and $\Gamma_{31}$ are proportional to $\tilde{g}$ and they identically vanish if $\tilde{g}=0$, as one can see in Fig. 2. Also, in this limit, $\Gamma_{11}$ and $\Gamma_{12}$ approach $\sin \alpha$ and $\cos \alpha$, respectively, where $\alpha$ is the usual CP-even Higgs mixing angle in the MSSM. (Notice that, despite in Fig. 2 the $\Gamma_{3 i}$ 's appear positive, they can in general take any sign, depending on the values of the mass matrix elements, being only subject to the sum rule $\sum_{i=1, \ldots 4}\left|\Gamma_{3 i}\right|^{2}=1$.)

The $h^{\prime}$ field can be written in terms of gauge eigenstates as

$$
h^{\prime}=\Gamma_{31} \sigma_{1}+\Gamma_{32} \sigma_{2}+\Gamma_{33} \sigma_{1}^{\prime}+\Gamma_{34} \sigma_{2}^{\prime} .
$$




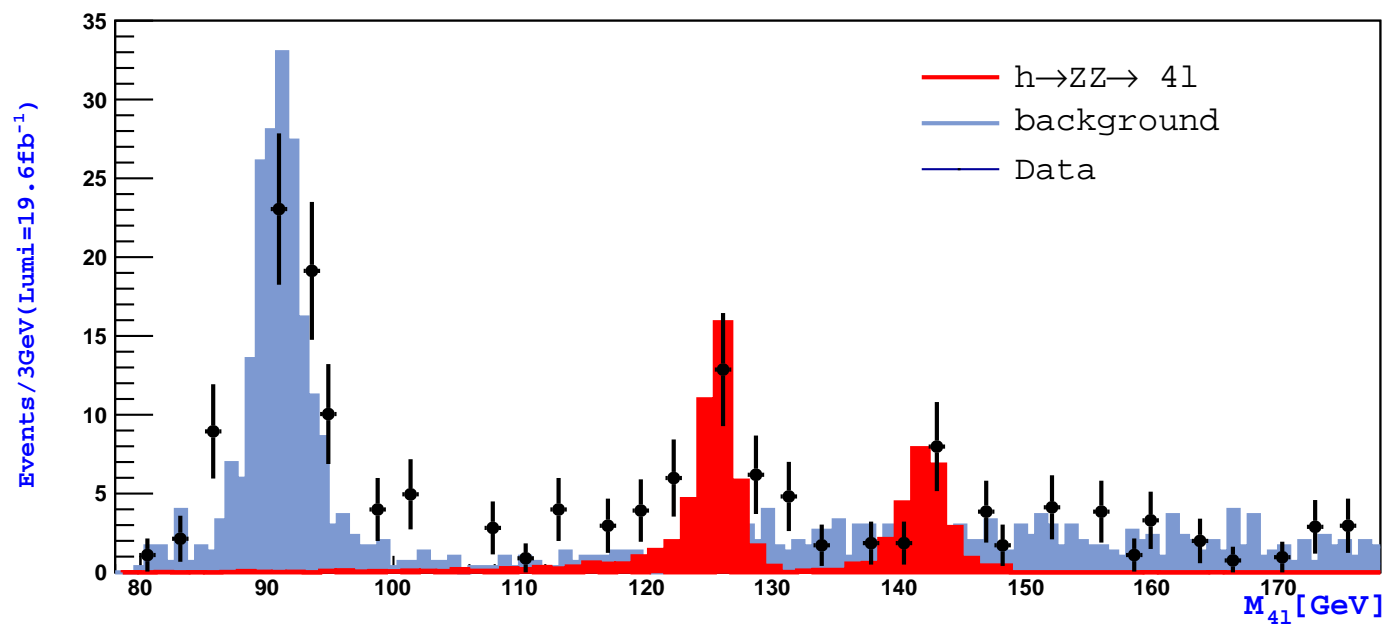

Figure 3. The number of events from the signal $p p \rightarrow h, h^{\prime} \rightarrow Z Z^{*} \rightarrow 4 l$ (red filled histogram) and from the background $p p \rightarrow Z \rightarrow 2 l \gamma^{*} \rightarrow 4 l$ (blue filled histogram) versus the invariant mass of the outgoing particles (4-leptons) against data (black dots) taken from [3].

Thus, the couplings of the $h^{\prime}$ with up- and down-quarks are given by

$$
h^{\prime} u \bar{u}:-i \frac{m_{u}}{v} \frac{\Gamma_{32}}{\sin \beta}, \quad h^{\prime} d \bar{d}:-i \frac{m_{d}}{v} \frac{\Gamma_{31}}{\cos \beta} .
$$

Similarly, one can derive the $h^{\prime}$ couplings with the $W^{+} W^{-}$and $Z Z$ gauge boson pairs:

$$
\begin{aligned}
h^{\prime} W^{+} W^{-} & : i g_{2} M_{W}\left(\Gamma_{32} \sin \beta+\Gamma_{31} \cos \beta\right), \\
h^{\prime} Z Z & : \frac{i}{2}\left[4 g_{B L} \sin ^{2} \theta^{\prime}\left(v_{1}^{\prime} \Gamma_{32}+v_{2}^{\prime} \Gamma_{31}\right)\right. \\
& \left.+\left(v_{2} \Gamma_{32}+v_{1} \Gamma_{31}\right)\left(g_{z} \cos \theta^{\prime}-\tilde{g} \sin \theta^{\prime}\right)^{2}\right] .
\end{aligned}
$$

Since $\sin \theta^{\prime} \ll 1$, the coupling of the $h^{\prime}$ with $Z Z, g_{h^{\prime} Z Z}$, will be as follows:

$$
g_{h^{\prime} Z Z} \simeq i g_{z} M_{Z}\left(\Gamma_{32} \sin \beta+\Gamma_{31} \cos \beta\right),
$$

where $g_{z}=\sqrt{g_{1}^{2}+g_{2}^{2}}$.

In the forthcoming numerical analysis we have used SARAH [16] and SPheno $[17,18]$ to build the BLSSM. Furthermore, the matrix-element calculation and event generation were derived from MadGraph 5 [19] and manipulated with MadAnalysis 5 [20]. Finally, notice that all current experimental constraints, from both collider (LEP2, Tevatron and LHC) and flavour (BaBar, Belle and LHCb) physics are taken into account in our numerical scans, by using (through SPheno) the programs HiggsBounds/HiggsSignals $[21,22]$ and SuperIso [23], respectively.

In Fig. 3 we show the invariant mass of the 4-lepton final state from $p p \rightarrow h^{\prime} \rightarrow Z Z^{*} \rightarrow 4 l$ as obtained at $\sqrt{s}=8 \mathrm{TeV}$ after $19.6 \mathrm{fb}^{-1}$ of luminosity and upon applying a $p_{T}$ cut of $5 \mathrm{GeV}$ on the four leptons. The SM contributions from the $Z$ and $125 \mathrm{GeV}$ Higgs boson decays, $p p \rightarrow Z \rightarrow 2 l \gamma^{*} \rightarrow 4 l$ and $p p \rightarrow h \rightarrow Z Z^{*} \rightarrow 4 l$, respectively, are taken into account too (with the same $p_{T}$ requirement), as demonstrated by the first two peaks in the plot. It is clear that the third peak at $m_{4 l} \approx 142 \mathrm{GeV}$, produced by the decay of the BLSSM Higgs boson $h^{\prime}$ into $Z Z^{*} \rightarrow 4 l$, can reasonably well account for the events observed by CMS [3].

The distribution of the di-photon invariant mass is presented in Fig. 4, again for a centre-of-mass energy of $\sqrt{s}=8 \mathrm{TeV}$ but now with a luminosity of $20.7 \mathrm{fb}^{-1}$. As previously, here too, the observed $h \rightarrow \gamma \gamma \mathrm{SM}$-like signal around $125 \mathrm{GeV}$ is shown (alongside the continuum) while the (rather subtle) 


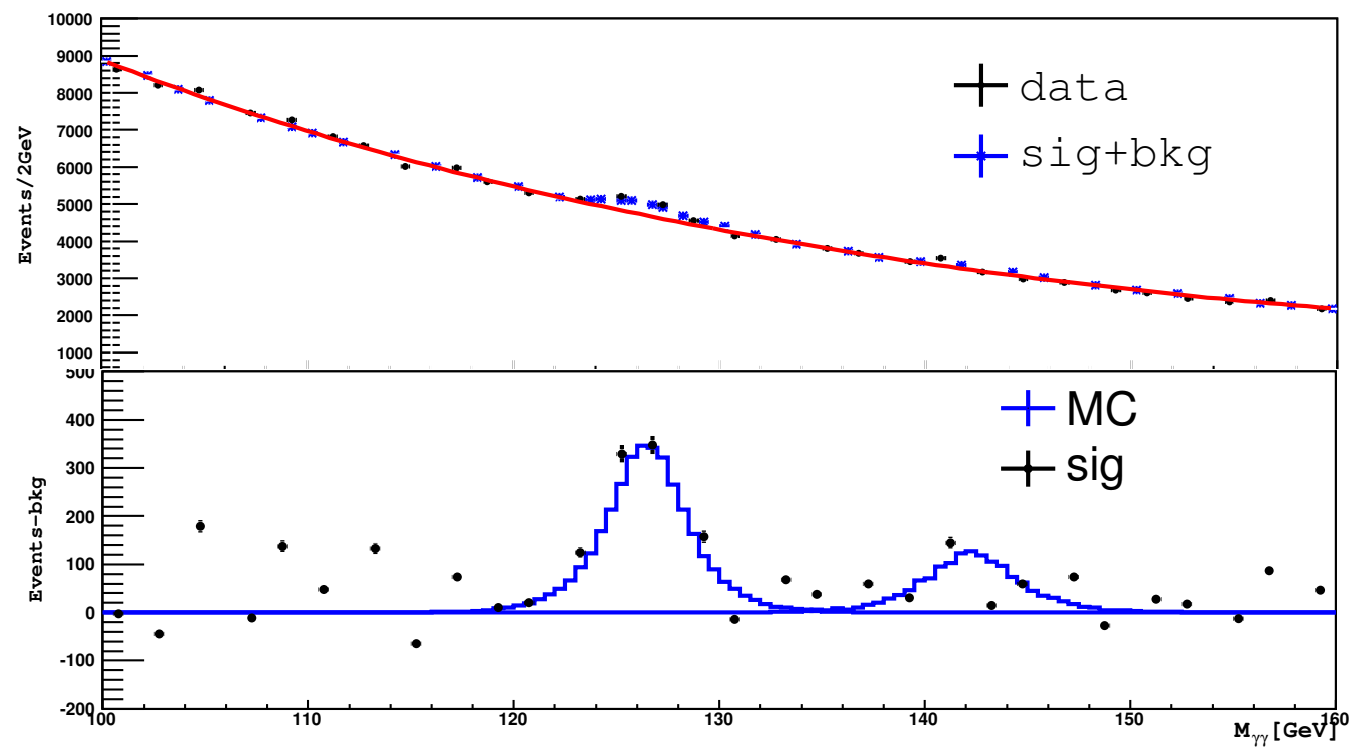

Figure 4. The number of events from the signal $p p \rightarrow h, h^{\prime} \rightarrow \gamma \gamma$ plus the background (blue points, top) and background $p p \rightarrow \gamma \gamma$ alone (red line, top) as well as the former without the latter (blue histogram, bottom) versus the invariant mass of the outgoing particles (di-photons) against data (black dots) taken from [5].

\begin{tabular}{|c|c|c|c|c|c|}
\hline \multicolumn{7}{|c|}{ Inputs } \\
\hline$g_{B L}$ & $\tilde{g}$ & $\tan \beta$ & $\tan \beta^{\prime}$ & $M_{Z^{\prime}}$ & $m_{H_{1}}^{2}$ \\
\hline 0.55 & 0.31 & 5 & 1.15 & 1700 & $1.1 \times 10^{6}$ \\
\hline$m_{H_{2}}^{2}$ & $m_{\chi_{1}}^{2}$ & $m_{\chi_{2}}^{2}$ & $Y_{\nu}^{\text {diag. }}$ & $Y_{N}^{\text {diag. }}$ & $\operatorname{sign}\left(\mu, \mu^{\prime}\right)$ \\
\hline$-1 \times 10^{7}$ & $-2.8 \times 10^{4}$ & $7.8 \times 10^{5}$ & $10^{-4}$ & 0.43 & 1 \\
\hline$\left(m_{\tilde{q}}^{2}\right)^{\text {diag. }}$ & $\left(m_{\tilde{\ell}}^{2}\right)^{\text {diag. }}$ & $\left(m_{\tilde{d}}^{2}\right)^{\text {diag. }}$ & $\left(m_{\tilde{u}}^{2}\right)^{\text {diag. }}$ & $\left(m_{\tilde{e}}^{2}\right)^{\text {diag. }}$ & $\left(m_{\nu}^{2}\right)^{\text {diag. }}$ \\
\hline $3.9 \times 10^{7}$ & $3.1 \times 10^{5}$ & $4 \times 10^{7}$ & $4 \times 10^{7}$ & $1.8 \times 10^{5}$ & $7.9 \times 10^{5}$ \\
\hline
\end{tabular}

Table 1. BLSSM benchmark point used in the analysis given in terms of relevant SARAH and SPheno inputs. (Dimensions of masses (squared) are $\mathrm{GeV}\left(\mathrm{GeV}^{2}\right)$.)

$Z^{*} \rightarrow \gamma \gamma$ background can now be ignored [24]. As expected, the sensitivity to the $h^{\prime}$ Higgs boson is severely reduced with respect to the presence of the already observed Higgs boson, yet a peak is clearly seen at $142 \mathrm{GeV}$ or so and is very compatible with the excess seen by ATLAS [5].

The BLSSM benchmark point used to simulate both the $Z Z^{*} \rightarrow 4 l$ and $\gamma \gamma$ signal is found in Tab. 1 .

Before closing, we should also mention that the $h^{\prime} \rightarrow \gamma \gamma$ enhancement found in the BLSSM may be mirrored in the $\gamma Z$ decay channel [15] for which, at present, there exists some constraints, albeit not as severe as in the $\gamma \gamma$ case.

Doubtless, if the second peak is real, explanations for it can be found in other SUSY models than just the BLSSM. Notable is the case of the Next-to-MSSM (NMSSM) [25,26], wherein the non-minimality is achieved by enlarging further the MSSM Higgs spectrum iself, by an additional Higgs singlet state, to elegantly obviate the so-called ' $\mu$-problem of the MSSM [27], another of its drawbacks. Further explanations can well be found within other SUSY realisations for the possible existence of a second peak. Far for judging the relative merits of each of these (and possibly other) SUSY scenarios, our intention was merely to alert the community that the hunt for Higgs bosons if far from over and that there may already be hints from data on where to find the next one.

It is now wait and see for a few more months, as no new Run 2 data for the di-photon invariant mass have been presented yet below $200 \mathrm{GeV}$. In fact, in the light of persisting anomalies from Run 1 
data in the 140-145 GeV region and our (as well as other) results, we advocate a combined analysis of all existing data (from both Run 1 and 2) in such mass region. The reward could be tantalising: not only the confirmation of a second scalar peak (i.e., of a non-minimal Higgs sector) but also in a mass region that would provide evidence (albeit circumstantial) of SUSY in a non-minimal form. As far as we are concerned, in fact, despite the potential benefits of a widespread Occam's razor attitude, we cannot disagree with the fact that "everything should be made as simple as possible, but not simpler", our favoured paraphrase of the Einstein's razor instead [28].

Acknowledgments. We thank A. Hammad for the plots. SK's work is partially supported from the STDF project 13858 while SM's is through the NExT Institute and the STFC Consolidated Grant $\mathrm{ST} / \mathrm{J000396/1}$. This research is also funded through the grant H2020-MSCA-RISE-2014 no. 645722 (NonMinimalHiggs).

\section{References}

1. F. Wilczek, Nature 496, 439 (2013).

2. I.I. Rabi, "Who ordered that?" (a quip in 1957, verbal).

3. CMS Collaboration, Phys. Rev. D 89, 092007 (2014).

4. CMS Collaboration, CMS-PAS-HIG-13-001 (2013) and CMS-PAS-HIG-13-016 (2013).

5. ATLAS Collaboration, ATLAS-CONF-2013-012.

6. ATLAS Collaboration, Phys. Rev. D 90, 112015 (2014).

7. CMS Collaboration, CMS-PAS-HIG-13-044 (2013); ATLAS Collaboration, ATLAS-CONF-2013-012 (2013) and Phys. Lett. B 726, 88 (2013) [Erratum, ibidem 734, 406 (2014)]; CDF \& D0 Collaborations, Phys. Rev. D 88, 052014 (2013).

8. ATLAS Collaboration, JHEP 1609, 1 (2016), JHEP 1609, 173 (2016), ATLAS-CONF-2016-059, ATLASCONF-2016-079, ATLAS-CONF-2016-082 and ATLAS-CONF-2016-083.

9. CMS Collaboration, CMS-PAS-HIG-16-001, CMS-PAS-HIG-16-014 and CMS-PAS-HIG-16-023.

10. CMS Collaboration, CMS-PAS-HIG-16-033.

11. A. Elsayed, S. Khalil and S. Moretti, Phys. Lett. B 715, 208 (2012).

12. S. Khalil and S. Moretti, Rep. Prog. Phys. 80, 036201 (2017).

13. B. O'Leary, W. Porod and F. Staub, JHEP 1205, 042 (2012).

14. W. Abdallah, S. Khalil and S. Moretti, Phys. Rev. D 91, 014001 (2015).

15. A. Hammad, S. Khalil and S. Moretti, Phys. Rev. D 92, 095008 (2015).

16. F. Staub, arXiv:0806.0538 [hep-ph].

17. W. Porod, Comput. Phys. Commun. 153, 275 (2003).

18. W. Porod and F. Staub, Comput. Phys. Commun. 183, 2458 (2012).

19. J. Alwall et al., JHEP 1407, 079 (2014).

20. E. Conte, B. Fuks and G. Serret, Comput. Phys. Commun. 184, 222 (2013).

21. P. Bechtle, O. Brein, S. Heinemeyer, O. Stål, T. Stefaniak, G. Weiglein and K. E. Williams, Comput. Phys. Commun. 181, 138 (2010) and Comput. Phys. Commun. 182, 2605 (2011).

22. P. Bechtle, S. Heinemeyer, O. Stål, T. Stefaniak and G. Weiglein, Eur. Phys. J. C 74, 2711 (2014).

23. F. Mahmoudi, Comput. Phys. Commun. 180, 1579 (2009).

24. S. Moretti, Phys. Rev. D 91, 014012 (2015).

25. G. Belanger, U. Ellwanger, J. F. Gunion, Y. Jiang, S. Kraml, arXiv:1208.4952 [hep-ph].

26. J. F. Gunion, Y. Jiang and S. Kraml, Phys. Rev. D 86, 071702 (2012).

27. U. Ellwanger, C. Hugonie and A. M. Teixeira, Phys. Rept. 496, 1 (2010).

28. A. Einstein, "On the Method of Theoretical Physics", The Herbert Spencer Lecture, delivered at Oxford (10 June 1933), published in Philosophy of Science, Vol. 1, No. 2 (April 1934), pp. 163-169, see p. 165. 